\title{
HILBERT-KUNZ FUNCTIONS FOR NORMAL RINGS
}

\author{
Craig Huneke, Moira A. McDermott, and Paul Monsky
}

ABSTRACT. Let $(R, \mathfrak{m}, k)$ be an excellent, local, normal ring of characteristic $p$ with a perfect residue field and $\operatorname{dim} R=d$. Let $M$ be a finitely generated $R$-module. We show that there exists $\beta(M) \in \mathbb{R}$ such that $\lambda\left(M / I^{[q]} M\right)=e_{H K}(M) q^{d}+$ $\beta(M) q^{d-1}+O\left(q^{d-2}\right)$.

\section{Introduction}

Throughout this paper $(R, \mathfrak{m})$ is a Noetherian local $\mathbb{Z} / p \mathbb{Z}$-algebra, $d=\operatorname{dim} R$, and $I \subset R$ is $\mathfrak{m}$-primary. We will let $n$ be a varying non-negative integer, and let $q=p^{n}$. By $I_{n}$ we will denote the ideal generated by $x^{q}, x \in I$. If $M$ is a finite $R$-module, $M / I_{n} M$ has finite length; we denote this length by $e_{n}(M, I)$, or more briefly by $e_{n}(M)$. We use $\lambda(-)$ to denote the length of an $R$-module. Let $f, g: \mathbb{N} \rightarrow \mathbb{R}$ be functions from the nonnegative integers to the real numbers. Recall that $f(n)=O(g(n))$ if there exists a positive constant $C$ such that $|f(n)| \leq C g(n)$ for all $n \gg 0$, and we write $f(n)=o(g(n))$ if $\lim _{n \rightarrow \infty} f(n) / g(n)=0$.

The basic question this paper studies is how $e_{n}(M)$ depends on $n$. The results of [Mo1] show that $e_{n}(M)=\alpha q^{d}+O\left(q^{d-1}\right)$ for some real $\alpha$. In section 1 we strengthen this proving:

Theorem 1. Let $(R, \mathfrak{m}, k)$ be an excellent, local, normal ring of characteristic $p$ with a perfect residue field and $\operatorname{dim} R=d$. Then $e_{n}(M)=\alpha q^{d}+\beta q^{d-1}+O\left(q^{d-2}\right)$ for some $\alpha$ and $\beta$ in $\mathbb{R}$.

In the situation of Theorem 1 it sometimes happens that $\beta(M)=0$ provided that $M=R$ (or more generally when $M$ is torsion-free). Our results establish that $\beta(M)=0$ whenever $M$ is torsion-free and the class group of $R$ is torsion. In particular this holds when $(R, \mathfrak{m}, k)$ is a complete normal two-dimensional ring and $k$ is finite-see Corollary 2.2.

One might hope that Theorem 1 could be generalized to prove that there exists a constant $\gamma$ such that $e_{n}(M)=\alpha q^{d}+\beta q^{d-1}+\gamma q^{d-2}+O\left(q^{d-3}\right)$ whenever $R$ is non-singular in codimension two. However, this cannot be true. For example,

Received September 8, 2003.

2000 Mathematics Subject Classification. Primary 13D40.

Key words and phrases. Hilbert-Kunz function.

The first author was partially supported by NSF grant DMS-0244405, and the second author was partially supported by the NSF-AWM Mentoring Travel Grant. We thank them for their support. 
if $R=\mathbb{Z} / 5 \mathbb{Z}\left[x_{1}, x_{2}, x_{3}, x_{4}\right] /\left(x_{1}^{4}+\cdots+x_{4}^{4}\right)$, then with $I=\left(x_{1}, \ldots, x_{4}\right), e_{n}(R)=$ $\frac{168}{61}\left(5^{3 n}\right)-\frac{107}{61}\left(3^{n}\right)$ by [HaMo]. Note that $R$ is a 3 -dimensional Gorenstein ring with isolated singularity. (Also see $[\mathrm{BuCh}]$ for computations of the Hilbert-Kunz function for plane cubics, as well as [Mo2]-[Mo4], [FaTr], and [Te] for other concrete computations of the Hilbert-Kunz function, and [WY1]-[WY3], [BE] for work on minimal possible values for the Hilbert-Kunz multiplicity.) It may however be true that $e_{n}(M)$ is always $\alpha q^{d}+\beta q^{d-1}+$ (periodic $)\left(q^{d-2}\right)+o\left(q^{d-2}\right)$. In particular we suspect that $e_{n}(M)=\alpha q^{2}+\beta q+$ (eventually periodic) when $d=2$. Striking results in this direction have recently been obtained by Brenner; see the footnote in section 2. See also [Co] for the case $A=k[[x, y]], I=(x, y)$, $M$ arbitrary.

\section{1.}

We make use of various facts about divisor classes in integrally closed Noetherian domains. Our reference is [Bo], and we shall need in particular Proposition 18 and Theorem 6 of chapter VII, section 4.

Let $R$ be an integrally closed Noetherian domain. A Weil divisor on $R$ is an element of the free abelian group on the height 1 primes of $R$. A principal Weil divisor is a divisor of the form $\sum_{P} \operatorname{ord}_{P}(f) \cdot P$ with $f \neq 0$ in the field of fractions of $R . C(R)$ is the quotient of the group of Weil divisors by the subgroup of principal divisors. Let $M$ be a finite $R$-module. Then $M$ admits a filtration with quotients (isomorphic to) $R / P_{i}$ where each $P_{i}$ is prime. Consider the Weil divisor $-\sum P_{i}$, the sum extending over those $P_{i}$ that are of height 1 . The image of this divisor in $C(R)$ is independent of the choice of filtration, and is denoted by $c(M)$. The map $c$ is additive on exact sequences and $c(R)=0$. If $P$ is a height 1 prime of $R$ the exact sequence $0 \rightarrow P \rightarrow R \rightarrow R / P \rightarrow 0$ shows that $c(P)=P$. Suppose now that we are in the situation of Theorem 1 of the introduction.

Lemma 1.1. Let $(R, \mathfrak{m}, k)$ be a local ring of characteristic $p$. If $T$ is a finitely generated torsion $R$-module with $\operatorname{dim} T=\ell$, then $\lambda\left(\operatorname{Tor}_{1}^{R}\left(R / I_{n}, T\right)\right)=O\left(q^{\ell}\right)$.

Proof. Set $d=\operatorname{dim} R$. Choose a system of parameters $\left(x_{1}, \ldots, x_{d}\right) \subseteq I$. We induct on $\lambda\left(I /\left(x_{1}, \ldots, x_{d}\right)\right)$. If $\lambda\left(I /\left(x_{1}, \ldots, x_{d}\right)\right)>0$, then there exists $J \subset I$ with $\lambda(I / J)=1$ so that we may write $I=(J, u)$ with $J: u=\mathfrak{m}$. For every $q=p^{n}$ there is an exact sequence

$$
0 \rightarrow R / J_{n}: u^{q} \rightarrow R / J_{n} \rightarrow R / I_{n} \rightarrow 0 .
$$

Tensor with $T$ and look at the following portion of the long exact sequence of Tors:

$$
\cdots \rightarrow \operatorname{Tor}_{1}^{R}\left(R / J_{n}, T\right) \rightarrow \operatorname{Tor}_{1}^{R}\left(R / I_{n}, T\right) \rightarrow \operatorname{Tor}_{0}^{R}\left(R / J_{n}: u^{q}, T\right) \rightarrow \cdots .
$$

We have $\lambda\left(\operatorname{Tor}_{1}^{R}\left(R / J_{n}, T\right)\right)=O\left(q^{\ell}\right)$ by induction. Also, since $J: u=\mathfrak{m}$, we have $\mathfrak{m}_{n} \subseteq J_{n}: u^{q}$ and $\lambda\left(\operatorname{Tor}_{0}^{R}\left(R / J_{n}: u^{q}, T\right)\right) \leq \lambda\left(\operatorname{Tor}_{0}^{R}\left(R / \mathfrak{m}_{n}, T\right)\right)$. But 
$\lambda\left(\operatorname{Tor}_{0}^{R}\left(R / \mathfrak{m}_{n}, T\right)\right)$ is the Hilbert-Kunz function for $T$, so $\lambda\left(\operatorname{Tor}_{0}^{R}\left(R / \mathfrak{m}_{n}, T\right)\right)=$ $O\left(q^{\ell}\right)$.

We have reduced to the case where $\lambda\left(I /\left(x_{1}, \ldots, x_{d}\right)\right)=0$. We need a theorem which is implicitly in Roberts [Ro] and explicitly given as Theorem 6.2 in [HH] (see also [Se, p278] for a theorem quickly giving an alternative proof with a sharper result on the growth of the size of the Koszul groups):

Theorem. Let $(R, m)$ be a local ring of characteristic $p$ and let $G$ • be a finite complex

$$
0 \rightarrow G_{n} \rightarrow \cdots \rightarrow G_{0} \rightarrow 0
$$

of length $n$ such that each $G_{i}$ is a finitely generated free module and suppose that each $H_{i}\left(G_{\bullet}\right)$ has finite length. Suppose that $M$ is a finitely generated $R$ module. Let $d=\operatorname{dim} M$. Then there is a constant $C>0$ such that $\ell\left(H_{n-t}\left(M \otimes_{R}\right.\right.$ $\left.F^{e}\left(G_{\bullet}\right)\right) \leq C q^{\min (d, t)}$ for all $t \geq 0$ and all $e \geq 0$, where $q=p^{e}$.

Consider $K_{\bullet}((\underline{x}) ; R)$, the Koszul complex on $\left(x_{1}, \ldots, x_{d}\right)$. Let $H_{\bullet}((\underline{x}) ; R)$ denote the homology of the Koszul complex. We apply the above theorem to conclude that there exists a constant $C>0$ such that $\lambda\left(H_{d-t}\left(T \otimes F^{n}\left(K_{\bullet}\right)\right)\right) \leq$ $C q^{\min (\ell, t)}$ for all $t$ and for all $n$. Hence $\lambda\left(H_{i}\left(T \otimes F^{n}\left(K_{\bullet}\right)\right)\right) \leq O\left(q^{\ell}\right)$ for all $i$. In general, $H_{1}\left(T \otimes F^{n}\left(K_{\bullet}\right)\right)$ maps onto $\operatorname{Tor}_{1}^{R}\left(T, R / I_{n}\right)$, which gives the stated result. (To see this, note that $F^{n}\left(K_{\bullet}\right)$ is exactly the Koszul complex on the generators of $I$ raised to the $q=p^{n}$ power, so that both of the complexes $F^{n}\left(K_{\bullet}\right)$ and the minimal free resolution of $R / I_{n}$ begin with the same two free modules.)

Lemma 1.2. Let $(R, \mathfrak{m}, k)$ be a local, normal ring of characteristic $p$. Let $J \neq$ (0) be an ideal of $R$. If $c(J)=0$ then $e_{n}(J)=e_{n}(R)+O\left(q^{d-2}\right)$.

Proof. The primary decomposition theorem shows that $J=\left(\cap P_{i}^{\left(n_{i}\right)}\right) \cap N$ where the $P_{i}^{\left(n_{i}\right)}$ are symbolic powers of finitely many height one primes $P_{i}$, and $\operatorname{dim}(R / N) \leq d-2$. Then $c(J)$ is the divisor class represented by $\sum n_{i} P_{i}$. So $\sum n_{i} P_{i}$ is the divisor of some $f \neq 0$ in $R$, and replacing $J$ by $f^{-1} J$ we may assume that $T=R / J$ is of dimension at most $d-2$. By Lemma 1.1, $\lambda\left(\operatorname{Tor}_{1}^{R}\left(T, R / I_{n}\right)\right)=$ $O\left(q^{d-2}\right)$ since $\operatorname{dim} T \leq d-2$. The exact sequence $\operatorname{Tor}_{1}^{R}\left(R / I_{n}, T\right) \rightarrow J / I_{n} J \rightarrow$ $R / I_{n} \rightarrow T / I_{n} T$ now gives the result, since $\lambda\left(T / I_{n} T\right)=O\left(q^{d-2}\right)$ by [Mo1].

Lemma 1.3. Let $(R, \mathfrak{m}, k)$ be a local, normal ring of characteristic $p$. Suppose $M$ is torsion-free of rank $r$ and $c(M)=0$. Then $e_{n}(M) \leq r e_{n}(R)+O\left(q^{d-2}\right)$.

Proof. By Theorem 6, section 4 of [Bo] there is an exact sequence $0 \rightarrow R^{r-1} \rightarrow$ $M \rightarrow J \rightarrow 0$ with $J$ an ideal. Then $c(J)=0$, and we use the exact sequence $\left(R / I_{n}\right)^{r-1} \rightarrow M / I_{n} M \rightarrow J / I_{n} J$ together with Lemma 1.2.

Theorem 1.4. Let $(R, \mathfrak{m}, k)$ be a local, normal ring of characteristic $p$. Suppose $M$ is torsion-free of rank $r$ and $c(M)=0$. Then $e_{n}(M)=r e_{n}(R)+O\left(q^{d-2}\right)$.

Proof. There is an exact sequence $0 \rightarrow K \rightarrow R^{r+s} \rightarrow M \rightarrow 0$ for some $K$ and $s \geq 0$. Then $K$ is torsion-free of rank $s$ and $c(K)=0$. By Lemma 1.3, 
$e_{n}(K) \leq s e_{n}(R)+O\left(q^{d-2}\right)$. Evidently $e_{n}(K)+e_{n}(M) \geq(r+s) e_{n}(R)$. So $e_{n}(M) \geq r e_{n}(R)+O\left(q^{d-2}\right)$; Lemma 1.3 provides the opposite inequality.

Lemma 1.5. Let $(R, \mathfrak{m}, k)$ be a local, normal ring of characteristic $p$. If $M$ is torsion-free, $\lambda\left(\operatorname{Tor}_{1}^{R}\left(R / I_{n}, M\right)\right)=O\left(q^{d-2}\right)$.

Proof. There is an exact sequence $0 \rightarrow K \rightarrow G \rightarrow M \rightarrow 0$ with $G$ free. Then $c(K \oplus M)=c(G)=0$, and Theorem 1.4 applied to $K \oplus M$ shows that $e_{n}(G)=$ $e_{n}(K)+e_{n}(M)+O\left(q^{d-2}\right)$. Now use the exact sequence $0 \rightarrow \operatorname{Tor}_{1}^{R}\left(R / I_{n}, M\right) \rightarrow$ $K / I_{n} K \rightarrow G / I_{n} G \rightarrow M / I_{n} M \rightarrow 0$.

Lemma 1.6. Let $(R, \mathfrak{m}, k)$ be a local, normal ring of characteristic $p$. Suppose $M$ and $N$ are torsion-free of the same rank and $c(M)=c(N)$. Then $e_{n}(M)=$ $e_{n}(N)+O\left(q^{d-2}\right)$.

Proof. Replacing $M$ and $N$ by $M \oplus J$ and $N \oplus J$ for some ideal $J$ we may assume that $c(M)=c(N)=0$. Now apply Theorem 1.4 to $M$ and to $N$.

Definition 1.7. Let $(R, \mathfrak{m}, k)$ be a local, normal ring of characteristic $p$. If $M$ is torsion-free of rank $r, \delta_{n}(M)=e_{n}(M)-r e_{n}(R)$.

Remarks. $\delta_{n}(R)=0$ and $\delta_{n}(M \oplus N)=\delta_{n}(M)+\delta_{n}(N)$. If $c(M)=c(N)$, Lemma 1.6 tells us that $\delta_{n}(M)=\delta_{n}(N)+O\left(q^{d-2}\right)$.

To make further progress we shall use the $p$ th power map $F: R \rightarrow R$, assuming $R$ is complete with perfect residue field. In this case $F$ is finite of degree $p^{d}$. Given a finite map $R \rightarrow R^{\prime}$ between integrally closed Noetherian domains, we obtain induced norm maps from Weil divisors on $R^{\prime}$ to Weil divisors on $R$ and from $C\left(R^{\prime}\right)$ to $C(R)$. For $F: R \rightarrow R$ we claim that these norm maps are just multiplication by $p^{d-1}$. For if $P$ is a height 1 prime of $R$, the only prime lying over $P$ is $P$ itself, and the ramification degree is evidently $p$. So the residue class field degree is $p^{d-1}$ by the discussion of Section 4.8, Chapter VII, page 535 in [Bo], and then the norm of $P$ is $p^{d-1} \cdot P$ by the same discussion.

If $M$ is a finitely generated $R$-module of rank $r$ let ${ }^{1} M$ be $M$ as additive group, but with $R$ acting through the $p$ th power map $F: R \rightarrow R$. Then ${ }^{1} M$ is evidently finite of rank $p^{d} r$, and $e_{n}\left({ }^{1} M\right)=e_{n+1}(M)$ for all $n$.

Theorem 1.8. Let $(R, \mathfrak{m}, k)$ be an excellent, local, normal ring of characteristic $p$ with a perfect residue field. Let $M$ be torsion-free of rank $r$. Then $\delta_{n+1}(M)=$ $p^{d-1} \delta_{n}(M)+O\left(q^{d-2}\right)$.

Proof. We may complete $R$ without changing the hypotheses or conclusions, and henceforth we assume that $R$ is complete. Since the norm map $C(R) \rightarrow C(R)$ induced by $F: R \rightarrow R$ is multiplication by $p^{d-1}$, Proposition 18 , section 4.8 , Chapter VII of [Bo] tells us that $c\left({ }^{1} M\right)=p^{d-1} c(M)+r c\left({ }^{1} R\right)$. The remarks after Definition 1.7 then show that $\delta_{n}\left({ }^{1} M\right)-r \delta_{n}\left({ }^{1} R\right)=p^{d-1} \delta_{n}(M)+O\left(q^{d-2}\right)$. But $\delta_{n}\left({ }^{1} M\right)-r \delta_{n}\left({ }^{1} R\right)=\left(e_{n+1}(M)-p^{d} r e_{n}(R)\right)-r\left(e_{n+1}(R)-p^{d} e_{n}(R)\right)=$ $e_{n+1}(M)-r e_{n+1}(R)=\delta_{n+1}(M)$, giving the theorem. 
Theorem 1.9. Let $(R, \mathfrak{m}, k)$ be an excellent, local, normal ring of characteristic $p$ with a perfect residue field. Let $M$ be a torsion-free finite $R$-module. There is a real constant $\tau(M)$ such that $\delta_{n}(M)=\tau(M) q^{d-1}+O\left(q^{d-2}\right)$.

Proof. Let $v_{n}=\delta_{n}(M) / q^{d-1}$. By Theorem 1.8, $v_{n+1}-v_{n}=O(1 / q)$. So $v_{n} \rightarrow$ some $\tau$, and $v_{n}=\tau+O(1 / q)$. The result follows.

Corollary 1.10. Let $(R, \mathfrak{m}, k)$ be an excellent, local, normal ring of characteristic $p$ with a perfect residue field. There is a homomorphism $\tau: C(R) \rightarrow \mathbb{R},+$ with the following property. If $M$ is torsion-free of rank $r$ then $e_{n}(M)=$ $r e_{n}(R)+\tau q^{d-1}+O\left(q^{d-2}\right)$ with $\tau=\tau(c(M))$.

Proof. If $c \in C(R)$ choose $M$ torsion-free with $c(M)=c$. Then $\delta_{n}(M)=$ $\tau q^{d-1}+O\left(q^{d-2}\right)$ for some real $\tau$. The remarks after Definition 1.7 tell us that $\tau$ is independent of the choice of $M$ and that $c \rightarrow \tau$ is a homomorphism.

We remark that it is immediate from this corollary that $\tau$ is the zero map whenever the class group of $R$ is torsion.

Theorem 1.11. Let $(R, \mathfrak{m}, k)$ be an excellent, local, normal ring of characteristic $p$ with a perfect residue field. Let $\operatorname{dim} R=d$. Then there exists $\beta(R) \in \mathbb{R}$ such that $e_{n}(R)=e_{H K}(I ; R) q^{d}+\beta(R) q^{d-1}+O\left(q^{d-2}\right)$. Furthermore, $\beta(R)\left(p^{d-1}-\right.$ $\left.p^{d}\right)=\tau\left({ }^{1} R\right)$.

Proof. Taking $M={ }^{1} R$ in Theorem 1.9 we find that $e_{n+1}(R)-p^{d} e_{n}(R)=\tau q^{d-1}+$ $O\left(q^{d-2}\right)$ where $\tau=\tau\left({ }^{1} R\right)$. Set $u_{n}=e_{n}(R)-\beta q^{d-1}$ where $\left(p^{d-1}-p^{d}\right) \beta=\tau$. Then $u_{n+1}-p^{d} u_{n}=e_{n+1}(R)-p^{d} e_{n}(R)-\tau q^{d-1}=O\left(q^{d-2}\right)$, and arguing as in the proof of Theorem 1.9 we find that $u_{n}=\alpha q^{d}+O\left(q^{d-2}\right)$.

In other words,

$$
e_{n}(R)=\alpha(R) q^{d}+\beta(R) q^{d-1}+O\left(q^{d-2}\right) \text { where } \beta(R)=\tau\left({ }^{1} R\right) /\left(p^{d-1}-p^{d}\right) .
$$

Clearly $\alpha(R)=e_{H K}(I ; R)$ is forced.

Theorem 1.12. Let $(R, \mathfrak{m}, k)$ be an excellent, local, normal ring of characteristic $p$ with a perfect residue field and $\operatorname{dim} R=d$. Let $M$ be finitely generated $R$-module. Then there exists $\beta(M) \in \mathbb{R}$ such that $e_{n}(M)=e_{H K}(I ; M) q^{d}+$ $\beta(M) q^{d-1}+O\left(q^{d-2}\right)$.

Proof. We again complete $R$ and assume it is complete. Suppose first that $M$ is torsion-free. Then the result follows from Theorems 1.9 and 1.11. In general there is an exact sequence $0 \rightarrow T \rightarrow M \rightarrow M^{\prime} \rightarrow 0$ with $T$ torsion and $M^{\prime}$ torsion free. The exact sequence $\operatorname{Tor}_{1}^{R}\left(R / I_{n}, M^{\prime}\right) \rightarrow T / I_{n} T \rightarrow M / I_{n} M \rightarrow M^{\prime} / I_{n} M^{\prime} \rightarrow$ 0 combined with Lemma 1.5 shows that $e_{n}(M)=e_{n}\left(M^{\prime}\right)+e_{n}(T)+O\left(q^{d-2}\right)$. Since $T$ has dimension $\leq d-1$, [Mo1] shows that $e_{n}(T)=c q^{d-1}+O\left(q^{d-2}\right)$ for some $c \geq 0$, and the result for $M^{\prime}$ yields the result for $M$.

A corollary of the above results gives us similar growth conditions on certain Tor modules. 
Corollary 1.13. Let $(R, \mathfrak{m}, k)$ be an excellent, local, normal ring of characteristic $p$ with perfect residue field and with $\operatorname{dim} R=d$. Let $T$ be a torsion $R$-module. Then there exists $\gamma(T) \in \mathbb{R}$ such that $\lambda\left(\operatorname{Tor}_{1}^{R}\left(T, R / I_{n}\right)\right)=\gamma(T) q^{d-1}+O\left(q^{d-2}\right)$.

Proof. We may complete $R$ and henceforth assume $R$ is complete. Consider an exact sequence,

$$
0 \rightarrow M \rightarrow R^{s} \rightarrow T \rightarrow 0
$$

where $M$ is torsion free. The long exact sequence on Tor after tensoring with $R / I_{n}$ shows that

$$
\lambda\left(\operatorname{Tor}_{1}^{R}\left(T, R / I_{n}\right)\right)=e_{n}(M)+e_{n}(T)-s e_{n}(R) .
$$

By [Mo1], $e_{n}(T)=c q^{d-1}+O\left(q^{d-2}\right)$ for some $c \geq 0$, while Theorem 1.9 shows that $e_{n}(M)-s e_{n}(R)=\tau(M) q^{d-1}+O\left(q^{d-2}\right)$. The corollary follows.

\section{The map $\tau$}

Given $(R, \mathfrak{m})$ and $I$ as in the last section, it might seem plausible that the map $\tau: C(R) \rightarrow \mathbb{R}$ is always the zero map, so that $e_{n}(M)=c q^{d}+O\left(q^{d-2}\right)$ for torsion-free $M$. This of course is true when $R$ is a UFD (or more generally when $C(R)$ is torsion), and so holds for $k\left[\left[x_{1}, \ldots, x_{r}\right]\right] /(F)$ when $F$ is a smooth form and $r$ is at least 5. However, K.-i. Watanabe has found counterexamples when $d$ is 3 or more. Here's a very nice example of his. Let $S$ be the $2 \times 3$ matrix whose entries are independent variables $x_{1}, \ldots, x_{6}$, and let $R$ be the quotient of $k\left[\left[x_{1}, \ldots, x_{6}\right]\right]$ by the ideal generated by the $2 \times 2$ minors of $S$. If $I=\mathfrak{m}$, then $e_{n}(R)=\left(13 q^{4}-2 q^{3}-q^{2}-2 q\right) / 8$. Hence $\beta(R)=-1 / 4$, and $\tau\left({ }^{1} R\right)=\left(p^{4}-p^{3}\right) / 4$. K. Kurano also commented on this point to us. He reports that $\beta(R)=0$ if the canonical class $c\left(K_{R}\right)$ is torsion in the divisor class group $C(R)$. (Here $R$ is local, excellent and normal.) His proof uses the singular Riemann-Roch theorem, and furthermore shows that if $R$ is Cohen-Macaulay and $I$ is a maximal primary ideal of finite projective dimension, then $e_{n}(R)$ is a polynomial in $q$ with rational coefficients. $^{1}$

When $d=2$ there are more general results. In particular the following Lemma seems to be known to experts, and we thank M. Artin and J. Lipman for pointing out relevant references and facts.

Lemma 2.1. Suppose that $(R, \mathfrak{m}, k)$ is a complete local normal two-dimensional ring, and $k$ is the algebraic closure of the field with $p$ elements. Then $C(R)$ is a torsion group.

\footnotetext{
${ }^{1} \mathrm{~A}$ recent preprint of $\mathrm{H}$. Brenner [Br1] shows that the Hilbert-Kunz multiplicity of the ring is rational in the two-dimensional graded case. This result was obtained independently by V. Trivedi [Tr]. In another more recent preprint [Br2] Brenner proves that $e_{n}(R)=\alpha q^{2}+$ an eventually periodic function of $n$ in the two-dimensional graded case over the algebraic closure of a finite field.
} 
Proof. The proof depends on the numerical theory of exceptional divisors (treated in full generality by Lipman), and arguments of Artin. An exposition is given by H. Göhner in [Gö], section 4, pages 423-426, which is independent of the rest of Göhner's paper. Note in particular the first part of Theorem 4.4 and corollary 4.5 in this paper. The hypothesis that there is a desingularization $f: X \rightarrow \operatorname{Spec}(R)$, made at the beginning of section 4 , is satisfied in this case, see [Li2].

Corollary 2.2. Suppose that $(R, \mathfrak{m}, k)$ is a complete local normal two-dimensional ring, and $k$ is finite. Then $\tau$ is the zero map.

Remark 2.3. For general algebraically closed $k$ there is an analog of Lemma 2.1. We adopt the notation of [Gö]. By $\left(^{*}\right)$ on page 425 there is an exact sequence

$$
0 \rightarrow \operatorname{Pic}^{0}(X) \rightarrow C(R) \rightarrow H \rightarrow 0
$$

with $H$ finite; see page 425 for the definition of $\operatorname{Pic}^{0}(X)$. To prove Lemma 2.1, Göhner uses Artin's result that there is a filtration of $\operatorname{Pic}^{0}(X)$ with each quotient isomorphic to either the additive group of $k, k^{*}$, or the group of $k$-valued points of the Jacobian variety of an irreducible component of the reduced special fibre of $f$. Somewhat more is true. There is a connected algebraic group $G$ defined over $k$, built out of copies of the additive group, the multiplicative group and the above Jacobians, such that $\operatorname{Pic}^{0}(X)$ identifies with $G_{k}$. For more information concerning this topic, see [Li1], in particular Theorem 7.5.

Remark 2.4. We believe that Corollary 2.2 holds even when $k$ is infinite. Here's an intuitive argument. Suppose that $P$ and $Q$ are in some sense "generic points" of $G_{k}=\operatorname{Pic}^{0}(X)$. Because the definition of $\tau$ is purely algebraic, $\tau(P)=\tau(Q)$. Since the various $P-Q$ with $P$ and $Q$ generic generate $G_{k}$, at least when $k$ is large enough, $\tau$ vanishes on the subgroup $\operatorname{Pic}^{0}(X)$ of $C(R)$ of finite index.

Remark 2.5. The third author has made the idea of the above remark into a simple proof when $R$ is the homogeneous coordinate ring of a smooth projective curve, localized at the homogeneous maximal ideal. In particular when $R=$ $k\left[\left[x_{1}, x_{2}, x_{3}\right]\right] /(F), F$ a smooth form, $\tau$ is the zero map. As we've noted this is also true for 5 or more variables-the 4 variable case remains open.

\section{References}

[BE] M. Blickle and F. Enescu. On rings with small Hilbert-Kunz functions. Proc. Amer. Math. Soc., 132 (2004), 2505-2509.

[Bo] N. Bourbaki. Elements of mathematics. Commutative algebra. Hermann, Paris, 1972. Translated from the French.

[Br1] H. Brenner. The rationality of the Hilbert-Kunz multiplicity in graded dimension two. Preprint, (2004), arXiv:math.AC/0402180.

[Br2] H. Brenner. The Hilbert-Kunz function in graded dimension two. Preprint, (2004), arXiv:math.AC/0405202.

[BuCh] R.-O. Buchweitz and Q. Chen. Hilbert-Kunz functions of cubic curves and surfaces. J. Algebra, 197 (1997), 246-267. 
[Co] M. Contessa. On the Hilbert-Kunz function and Koszul homology. J. Algebra, 175 (1995), 757-766.

[FaTr] N. Fakhruddin and V. Trivedi. Hilbert-Kunz functions and multiplicities for full flag varieties and elliptic curves. J. Pure Appl. Algebra, 181 (2003), 23-52.

[Gö] H. Göhner. Semifactoriality and Muhly's condition (N) in two dimensional local rings. J. Algebra, 34 (1975), 403-429.

[HH] M. Hochster and C. Huneke. Phantom homology. Mem. Amer. Math. Soc., 103 (1993).

[HaMo] C. Han and P. Monsky. Some surprising Hilbert-Kunz functions. Math. Z., 214 (1993), 119-135.

[Li1] J. Lipman. The Picard group of a scheme over an Artin ring. Inst. Hautes Études Sci. Publ. Math., 46 (1976), 15-86.

[Li2] Desingularization of two-dimensional schemes. Annals of Math., 107 (1978), 151-207.

[Mo1] P. Monsky. The Hilbert-Kunz function. Math. Ann., 263 (1983), 43-49.

[Mo2] The Hilbert-Kunz function of a characteristic 2 cubic. J. Algebra, 197 (1997), 268-277.

[Mo3] Hilbert-Kunz functions in a family: point- $S_{4}$ quartics. J. Algebra, 208 (1998), 343-358.

[Mo4] Hilbert-Kunz functions in a family: line- $S_{4}$ quartics. J. Algebra, 208 (1998), 359-371.

[Ro] P. Roberts. Le théorème d'intersection. C. R. Acad. Sci. Paris Sér. I Math., 304 (1987), 177-180.

[Se] G. Seibert. Complexes with homology of finite length and Frobenius functors. $J$. Algebra, 125 (1989), 278-287.

[Te] P. Teixeira. $p$-fractals and Hilbert-Kunz series. Ph.D. thesis, Brandeis University, 2002.

[Tr] V. Trivedi. Semistability and Hilbert-Kunz multiplicity for curves. Preprint, (2004), arXiv:math.AC/0402245.

[WY1] K.-i. Watanabe and K.-i. Yoshida. Hilbert-Kunz multiplicity and an inequality between multiplicity and colength. J. Algebra, 230 (2000), 295-317.

[WY2] Hilbert-Kunz multiplicity of two-dimensional local rings. Nagoya Math. J., 162 (2001), 87-110.

[WY3] Hilbert-Kunz multiplicity of three-dimensional local rings. Preprint, (2003), arXiv:math.AC/0307294.

Department of Mathematics, University of Kansas, Lawrence, KS 66045

E-mail address: huneke@math.ukans.edu

$U R L:$ http://www. math.ukans.edu/ huneke

Department of Mathematics and Computer Science, Gustavus Adolphus College, SAINT PETER, MN 56082

E-mail address: mmcdermo@gac.edu

$U R L:$ http: //www.gustavus.edu/ ${ }^{\text {mmcdermo }}$

Department of Mathematics, Brandeis University, Waltham, MA 02254-9110

E-mail address: monsky@brandeis.edu

$U R L:$ http://www . math. brandeis.edu/homepages/monsky 